\title{
Durcissement par seconde phase dans les aciers
}

\author{
JEAN-HuBERT SchmitT ${ }^{a, b}$ \\ ARCELOR Innovation R\&D, UGINE\&ALZ - CRI, BP 15, 62330 Isbergues, France
}

Reçu le 10 juin 2003, accepté le 7 novembre 2003

Résumé - Selon la composition chimique et la température, les aciers présentent deux phases à l'équilibre : austénite et ferrite. La solubilité quasi nulle du carbone et de l'azote dans la ferrite à température ambiante favorise la précipitation de carbures et/ou de nitrures pouvant conduire au durcissement de la matrice. Pour les fortes teneurs en carbone, apparaît une phase eutectoïde dure, la perlite, constituée de lamelles de ferrite et de cémentite (carbure de fer). Les aciers ferrito-perlitiques (matrice ferritique avec des îlots de perlite) présentent une limite d'élasticité élevée avec des potentialités d'allongement importantes ; un durcissement complémentaire peut être obtenu par écrouissage (par exemple, les aciers steel cord). Un refroidissement rapide de l'austénitique conduit à des phases métastables dures : la martensite et la bainite. Selon la teneur en carbone et le schéma de refroidissement, on obtient généralement des structures multiphasées à matrice ferritique; la transformation martensitique se produisant avec un changement de volume, la limite d'élasticité est affectée par la formation de ce constituant. Dans certains cas particuliers, l'austénite résiduelle après refroidissement se transforme progressivement en martensite durant la déformation. Cet effet TRIP (Transformation Induced Plasticity - Transformation induite par la plasticité) permet l'obtention d'une résistance élevée avec une ductilité importante. Ainsi, il est possible d'obtenir différentes familles d'acier couvrant un large domaine d'utilisation. La modélisation de leur comportement mécanique doit permettre de définir les structures optimales pour une tenue mécanique visée.

Mots clés : Aciers / multiphasés / plasticité / durcissement / structure

Abstract - Second phase hardening in carbon steel. According to the chemical composition of steel and the temperature, two different phases exist: namely, austenite and ferrite. The carbon and nitrogen solubility is very low at room temperature in the ferrite; it favors the precipitation of carbides and/or nitrides, increasing the mechanical resistance of the steel. At higher carbon content, a hard eutectoid phase appears, the pearlite, an alternation of ferrite and cementite (iron carbide) lamellae. The ferrite-pearlite steels (pearlite islands dispersed within the ferrite matrix) exhibit a high yield stress combined with a large elongation; the alloy can be subsequently strain-hardened (e.g. steel-cord wires). A rapid cooling from the austenite state leads to metastable hard phases such as martensite and bainite. Depending on the carbon content and the cooling rate, a multicomponent structure can be obtained with a ferritic matrix: e.g. dual-phase steel structure. As the martensite transformation occurs with a volume change, the yield stress is directly influenced by the appearance of this constituent. In some specific conditions, residual austenite, which is metastable at the end of the cooling, transforms progressively in martensite during a subsequent deformation. This TRIP (Transformation Induced Plasticity) effect gives rise to a high tensile strength combined with a large ductility. Many different steels can thus be obtained for a wide range of mechanical properties. The modeling of their mechanical behavior will be a help for defining the optimal structures to achieve various mechanical properties.

Key words: Steels / dual phase / plasticity / hardening / substructure

\footnotetext{
a Auteur correspondant : schmitt@ads.ecp.fr

b Nouvelle adresse : École centrale Paris, Grande voie des Vignes, 92295 Châtenay-Malabry Cedex, France
} 


\section{Nomenclature}

\begin{tabular}{|c|c|c|}
\hline$b$ & norme du vecteur de Burgers des dislocations dans la ferrite & $\mathrm{m}$ \\
\hline$D$ & taille de grain & $\mathrm{m}$ \\
\hline$k$ & $\begin{array}{l}\text { coefficient de proportionnalité dans l'expression du libre parcours moyen } \\
\text { des dislocations (de l'ordre de quelques unités) }\end{array}$ & \\
\hline$k_{p}$ & coefficient de type Hall-Petch, rendant compte de l'influence & \\
\hline & de l'espacement interlamellaire perlitique & МРa. $\mu \mathrm{m}^{1 / 2}$ \\
\hline$f$ & $\begin{array}{l}\text { coefficient de proportionnalité dans l'expression de l'annihilation des dislocations } \\
\text { (de l'ordre de } 5 \text { ) }\end{array}$ & \\
\hline$F_{p}$ & fraction volumique de perlite & \\
\hline$S$ & distance interlamellaire dans la perlite & $\mu \mathrm{m}$ \\
\hline$\alpha$ & $\begin{array}{l}\text { coefficient de proportionnalité reliant la contrainte à la densité de dislocation } \\
\text { (de l'ordre de l'unité) }\end{array}$ & \\
\hline$\varepsilon$ & déformation plastique imposée & \\
\hline$\varepsilon_{\alpha}$ & déformation dans la ferrite pour une déformation moyenne $\varepsilon$ dans le matériau biphasé & \\
\hline$\varepsilon_{p}$ & déformation dans la perlite pour une déformation moyenne $\varepsilon$ dans le matériau biphasé & \\
\hline$\mu$ & module de cisaillement & $\mathrm{MPa}$ \\
\hline$\rho$ & densité de dislocations dues à la déformation plastique & $\mathrm{m} \cdot \mathrm{m}^{-3}$ \\
\hline$\sigma_{p}$ & contrainte d'écoulement dans la perlite & $\mathrm{MPa}$ \\
\hline$\sigma_{p_{y}}$ & limite d'élasticité dans la perlite & $\mathrm{MPa}$ \\
\hline$\sigma_{p_{o}}$ & contribution de la ferrite à la limite d'élasticité de la perlite & $\mathrm{MPa}$ \\
\hline$\sigma_{\alpha}$ & contrainte d'écoulement dans la ferrite & $\mathrm{MPa}$ \\
\hline$\sigma_{\alpha_{o}}$ & $\begin{array}{l}\text { contrainte d'écoulement résultant du frottement de réseau, des éléments en solution solide } \\
\text { et de la précipitation }\end{array}$ & $\mathrm{MPa}$ \\
\hline$\sigma(\varepsilon)$ & contrainte d'écoulement du matériau biphasé ferrite-perlite pour une déformation $\varepsilon$ donnée & $\mathrm{MPa}$ \\
\hline
\end{tabular}

\section{Introduction}

Les aciers, alliages à base fer-carbone, présentent deux particularités qui offrent les possibilités d'une métallurgie «flexible»: (i) l'existence de deux phases, austénite et ferrite, (ii) la présence d'un eutectoïde (fer-cémentite). Ces spécificités conduisent à l'obtention de microstructures très variées en fonction de la composition chimique de l'alliage et des traitements thermomécaniques imposés. Il est ainsi possible d'obtenir des familles d'aciers à propriétés mécaniques étendues, particulièrement dans le domaine des hautes et très hautes résistances (la contrainte maximale à rupture peut varier de $200 \mathrm{MPa}$ à $1500 \mathrm{MPa}$, voire plus en jouant sur l'écrouissage du produit final).

(i) L'austénite, constituant de structure cubique à faces centrées, est généralement stable à haute température. Son domaine d'existence dépend de la composition chimique de l'alliage ; dans certains cas, ce constituant peut être stable (ou méta-stable) à la température ambiante.

La ferrite, de structure cubique centrée, existe à l'ambiante; elle se distingue en particulier par une très faible solubilité des éléments interstitiels comme le carbone ou l'azote. Cette caractéristique conduit à une précipitation très rapide de carbures (et/ou carbonitrures et/ou carbosulfures) lors du refroidissement d'un acier ou à l'apparition de constituants méta-stables présentant une sursaturation en carbone (martensite, bainite). (ii) L'existence d'un eutectoïde (pour 0,77 \% en poids de carbone à $727{ }^{\circ} \mathrm{C}$ pour l'alliage binaire fer-carbone ${ }^{1}$ ) permet l'obtention d'un composé : la perlite, formée de ferrite et de cémentite (carbure de fer, $\mathrm{Fe}_{3} \mathrm{C}$ ). $\mathrm{Ce}$ composé est une structure biphasée dont nous verrons ultérieurement les particularités.

Différents types de structures sont obtenues à partir d'une matrice ferritique et d'une dispersion d'un second constituant : perlite, bainite ou martensite, voire, de façon simultanée, de plusieurs de ces constituants. Dans cette présentation, nous décrivons comment obtenir certaines de ces structures multiphasées dans les aciers, nous évoquons succinctement leurs principales caractéristiques, propriétés et utilisations, et nous concluons par l'évocation des problèmes posés par la modélisation de leur comportement mécanique.

\section{La cémentite : un composé lamellaire}

\subsection{Formation et structure de la perlite}

$\mathrm{Au}$ cours d'un refroidissement à vitesse lente (inférieure à quelques dizaines de $\mathrm{K} . \mathrm{s}^{-1}$ ), l'austénite se décompose en un mélange de ferrite proeutectö̈de et de

\footnotetext{
${ }^{1}$ Ces valeurs sont des fonctions des éléments d'addition présents dans l'alliage, en particulier de la teneur en chrome.
} 
perlite. La fraction volumique de perlite varie de zéro pour-cent dans les aciers extra-doux (teneur en carbone inférieure à $0,05 \%$ en poids) à $100 \%$ dans les aciers à $0,77 \%$ en poids de carbone. Lorsque la teneur en carbone est supérieure (aciers hypereutectö̈des), la phase proeutectoïde est la cémentite.

La perlite se forme par germination simultanée des deux phases lors de la transformation de l'austénite. Le plus souvent le mélange se présente localement sous forme de lamelles juxtaposées de ferrite et de cémentite ${ }^{2}$. Pour respecter les concentrations d'équilibre en carbone dans chaque phase, l'épaisseur des lamelles de cémentite est environ 6 fois plus faible que celle des lamelles de ferrite. Les caractéristiques thermiques du refroidissement (vitesse de refroidissement, température de transformation, ...) modifient simultanément ces deux paramètres structuraux. L'espacement interlamellaire (distance entre deux lamelles de cémentite voisines) est d'autant plus petit que la température de transformation est plus basse. Dans la pratique, cette distance se situe généralement entre 0,1 et $1 \mu \mathrm{m}$.

Des relations cristallographiques particulières assurent la correspondance entre les réseaux cubique de la ferrite $(\alpha)$ et orthorhombique de la cémentite. Différentes relations sont proposées dans la littérature, mais on retient usuellement celles de Bagaryatsky [1] :

$$
\begin{aligned}
& {[100]_{\mathrm{Fe}_{3} \mathrm{C}} / /\langle 1 \overline{1} 0\rangle_{\alpha}} \\
& {[010]_{\mathrm{Fe}_{3} \mathrm{C}} / /\langle 111\rangle_{\alpha}} \\
& (001)_{\mathrm{Fe}_{3} \mathrm{C}} / /\{11 \overline{2}\}_{\alpha}
\end{aligned}
$$

Cette relation a un impact direct sur la déformation de la perlite dans la mesure où le plan d'accolement $\left((001)_{\mathrm{Fe}_{3} \mathrm{C}}\right)$ est parallèle à un plan de glissement possible dans la ferrite.

D'autre part, pour une même orientation d'un grain de ferrite, il peut exister plusieurs variants de cémentite conduisant à la présence de colonies de perlite [2] : même orientation cristallographique de la ferrite et orientations différentes des lamelles de cémentite (Fig. 1). Ces colonies sont issues d'un même grain d'austénite à haute température. Les zones de transition entre les colonies sont des domaines d'accommodation possible de la déformation plastique.

\subsection{Déformation de la perlite}

La perlite se comporte comme un composite lamellaire. La différence de comportement mécanique est très importante entre les deux phases et la déformation débute dans la matrice ferritique.

Dans le cas des espacements interlamellaires importants (supérieur au micromètre), la déformation au sein

\footnotetext{
${ }^{2}$ Un revenu prolongé à haute température conduit à la globulisation de la perlite. Des particules sphéroïdales de cémentite sont dispersées au sein d'une matrice ferritique. La structure et le comportement sont alors similaires à ceux d'un matériau avec des inclusions ou un renfort de particules.
}

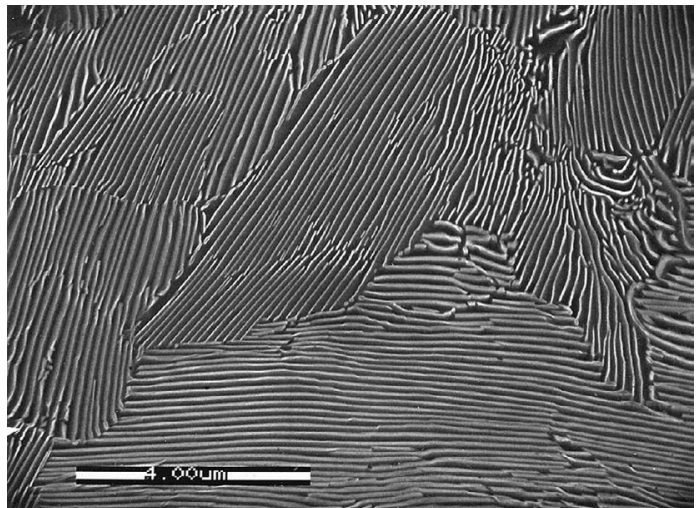

Fig. 1. Image MEB en électrons secondaires de colonies perlitiques (ferrite en noir et cémentite en clair) - la barre d'échelle correspond à $4 \mu \mathrm{m}$.

des lamelles de ferrite est similaire à celle d'un acier totalement ferritique : très rapidement le libre parcours moyen des dislocations devient plus faible que la distance interlamellaire et, pour les forts taux de déformation, des cellules de dislocations se développent entre les lamelles de cémentite [3].

Lorsque l'espacement interlamellaire est faible (de l'ordre du dixième de micromètre), les dislocations sont « canalisées » entre les lamelles de cémentite comme cela a été observé in situ en microscopie électronique en transmission par Janecek et al. [4].

Dans tous les cas, les zones de transition entre les colonies jouent un rôle important : de nombreuses dislocations viennent se stocker, induisant une désorientation progressive de la ferrite entre les différentes colonies. Ce mécanisme particulier peut s'accompagner du phénomène de curling lors de déformations importantes par tréfilage (le grain, initialement équiaxe, s'incurve dans la section du fil pour accommoder une part de la déformation imposée par une rotation solide).

Enfin, bien que fragile, la cémentite peut cependant se déformer plastiquement, d'autant plus lorsqu'une pression hydrostatique est imposée au matériau (cas, par exemple, du tréfilage où des taux de déformation de 3 ou 4 peuvent alors être atteints). Il a été montré que la cémentite se déforme par glissement localisé de dislocations avec un taux de stockage très faible (donc un très faible écrouissage lors de la déformation) [2].

Même si les mécanismes physiques de la déformation n'ont pas été complètement compris et modélisés, des relations empiriques sont proposées afin de rendre compte du durcissement de la perlite. D’une façon générale, l'espacement interlamellaire influence au premier ordre la limite d'élasticité de la perlite. Par analogie avec la relation de Hall-Petch, il a été proposé, entre autres par Pickering [5], d'exprimer la limite d'élasticité de la perlite, $\sigma_{p_{y}}$, en fonction de la distance interlamellaire, $S$ :

$$
\sigma_{p_{y}}=\sigma_{p_{o}}+\frac{k_{p}}{\sqrt{S}}
$$




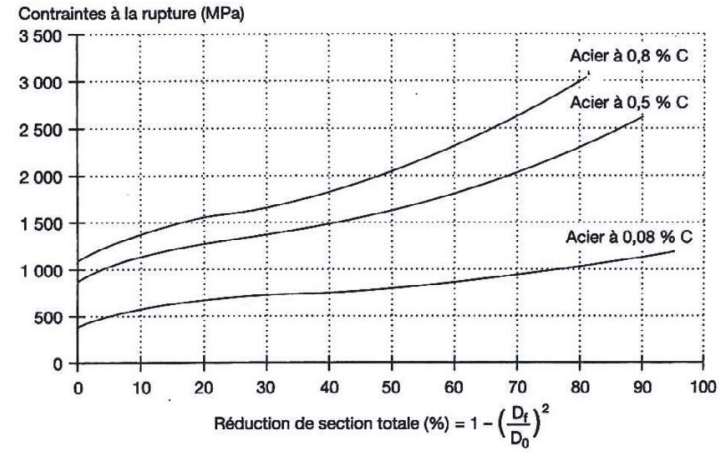

Fig. 2. Évolution de la résistance d'un fil perlitique $(0,8 \% \mathrm{C})$ ou ferrito-perlitique lors du tréfilage.

où $\sigma_{p_{o}}$ est fonction de la composition et de l'état de précipitation de la ferrite.

Dans les cas pratiques, on mesure des valeurs de limite d'élasticité de l'ordre de $450 \mathrm{MPa}$ pour une distance interlamellaire de $1 \mu \mathrm{m}$, et de $550 \mathrm{MPa}$ pour une valeur de $S$ de $0,1 \mu \mathrm{m}$.

La structure perlitique présente une déformabilité très importante lors d'un tréfilage [6]. Cette déformation s'accompagne d'un écrouissage continu et conduit à des résistances supérieures à $3000 \mathrm{MPa}$ (Fig. 2). Cette caractéristique est utilisée pour obtenir des fils fins ultrarésistants servant de renforts dans les pneumatiques (steel cord).

Lors de cette déformation, l'espace interlamellaire diminue (en moyenne, proportionnellement à la réduction de section) et les lamelles tendent à s'aligner dans la direction de tréfilage. Pour les déformations extrêmes, l'épaisseur des lamelles de cémentite devient si faible que cette phase tend à disparaître par remise en solution de la cémentite [3].

\section{Les structures de trempe}

La perlite est un exemple très particulier des structures biphasées dans les aciers. Beaucoup plus couramment, les aciers à haute résistance présentent une matrice ferritique durcie par une dispersion d'un second constituant. Avant de présenter plus en détail ces structures et leurs caractéristiques, il est nécessaire de décrire brièvement quelques exemples de ces constituants durcissants. Comme évoqué dans l'introduction, ceux-ci sont, le plus souvent, des structures de trempe, c'est-à-dire des structures hors d'équilibre provenant du refroidissement rapide de l'austénite.

\subsection{Martensite et bainites}

Pour des vitesses de refroidissement de l'austénite supérieures à une vitesse critique qui dépend de la composition chimique de l'alliage et de la structure de l'austénite (taille de grain, écrouissage résiduel, ...), on obtient,

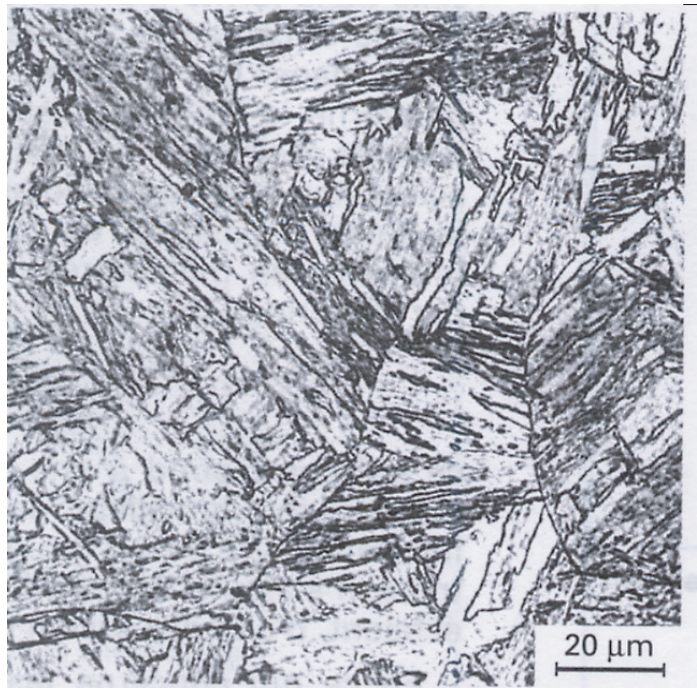

Fig. 3. Exemple de structure martensitique en lattes.

en dessous d'une température $\mathrm{M}_{\mathrm{S}}$ [5], un constituant métastable : la martensite (Fig. 3).

La martensite, de structure tétragonale centrée, dérive de la structure cubique centrée de la ferrite et se forme à partir de l'austénite par une transformation à caractère displacif sans diffusion à longue distance $[7,8]$. La fraction volumique de martensite formée est uniquement fonction de la température de fin de refroidissement et atteint $100 \%$ pour une température $\mathrm{M}_{\mathrm{f}}$; cette fraction n'évolue pas avec le temps de maintien à une température donnée entre $\mathrm{M}_{\mathrm{s}}$ et $\mathrm{M}_{\mathrm{f}}$. Pour des teneurs en carbone inférieures à $0,5 \%$ en poids, la martensite se présente sous forme de lattes [9], se formant généralement à partir des joints de grain de l'austénite et se propageant vers l'intérieur du grain. Ces lattes, d'une épaisseur de l'ordre du dixième de micromètre et d'une longueur de quelques micromètres, sont regroupées en paquet; au sein d'un paquet, la désorientation cristalline entre les lattes est faible, alors qu'elle peut atteindre plusieurs dizaines de degrés entre les paquets.

La dureté de ce constituant est directement liée à la sursaturation en carbone et d'autant plus forte que la teneur en carbone est importante. Elle résulte également de la présence d'une forte densité de dislocations d'accommodation aux interfaces entre lattes.

Lorsque la vitesse de refroidissement depuis l'état austénitique est intermédiaire entre celle de la transformation perlitique et celle de la transformation martensitique, il se forme divers constituants appelés bainites. D'une façon générale, la microstructure de ces constituants est un mélange non lamellaire de ferrite et de cémentite. On distingue différentes classes de bainite en fonction de la répartition des particules de cémentite dans la microstructure (pour plus de détails, voir [10]).

- La bainite supérieure est constituée de paquets de lattes ferritiques parallèles avec des particules de cémentite décorant les joints de lattes. Ce constituant 
se forme pour des températures de transformation plus élevées.

- La structure ferritique de la bainite inférieure est similaire, mais la précipitation de cémentite s'effectue à l'intérieur des lattes sous forme de fines plaquettes ou d'aiguilles.

Dans les deux cas, plus la vitesse de refroidissement est grande, plus les lattes sont fines. Les désorientations cristallines sont faibles entre les lattes d'un même paquet (au maximum quelques degrés) et de l'ordre de quelques dizaines de degrés entre deux paquets de lattes. Il se constitue ainsi des unités microstructurales dont l'impact est fort sur les propriétés mécaniques, en particulier dans ce cas, sur la ténacité [11].

D'une manière générale, la martensite et la bainite sont des constituants durs et fragiles. On les trouve le plus souvent associés à une matrice ferritique, formant ainsi une structure biphasée (aciers dual phase) ferritomartensitique ou ferrito-bainitique.

\subsection{Martensite induite}

Pour des températures légèrement supérieures à $M_{S}$, une déformation plastique de l'austénite peut induire une transformation martensitique. Ce mécanisme est décrit sous le nom d'effet TRIP (Transformation Induced Plasticity). Il est fortement influencé par l'énergie de faute d'empilement de l'alliage, c'est-à-dire par sa composition chimique. Une température $\mathrm{M}_{\mathrm{d}}$ a été définie de façon empirique permettant de mesurer la sensibilité d'un alliage à l'effet TRIP ; cette température correspond à la formation de $50 \%$ de martensite après $30 \%$ de déformation en traction. Les teneurs en carbone et en azote en solution solide dans l'austénite influencent très fortement cette température [5].

En ajustant la composition de l'alliage, il est possible de conserver une austénite métastable à température ambiante, c'est-à-dire se transformant progressivement en martensite lors de la déformation. Cette transformation contribue au durcissement progressif du métal et s'ajoute à l'écrouissage de l'austénite : la ductilité résultante en est fortement accrue. On observe alors une évolution de la microstructure avec une modification de la nature et du comportement mécanique de la martensite.

Dans les aciers au carbone, les conditions de refroidissement sont ajustées afin d'accroître la teneur en carbone dans l'austénite résiduelle pour la stabiliser à température ambiante. On forme alors un acier TRIP, c'est-à-dire une matrice ferritique (ou ferrito-bainitique) avec des îlots d'austénite résiduelle.

\section{Aciers biphasés}

La demande croissante en produits formables à haute résistance conduit au développement rapide d'aciers biet multiphasés. Cette voie permet en effet de combiner la déformabilité associée à une matrice ferritique au durcissement d'une seconde phase. La recherche d'une diminution des coûts et d'un bon comportement en soudage impose de limiter les ajouts d'éléments d'addition. De plus en plus, les microstructures complexes sont obtenues pour des aciers faiblement alliés en contrôlant les schémas de refroidissement après le laminage à chaud et/ou après le recuit des tôles laminées à froid.

\subsection{Voies d'obtention des structures biphasées}

Dans les nuances à faible ou moyenne teneur en carbone, la transformation de phase débute par la formation de ferrite aux joints des grains austénitiques, principalement aux joints triples. Du fait de la très faible solubilité du carbone dans la ferrite, l'austénite s'enrichit en carbone au fur et à mesure du refroidissement [12].

\subsubsection{Aciers ferrito-perlitiques}

Si la vitesse de refroidissement est relativement lente, on atteint une concentration en carbone dans l'austénite telle que la transformation perlitique peut s'amorcer. On obtient alors une structure biphasée ferrite-perlite, courante pour les aciers à moyenne teneur en carbone utilisés pour la construction mécanique. Le durcissement de ces aciers reste modéré.

La matrice ferritique peut être durcie de façon complémentaire par l'addition d'éléments en solution solide ou par une fine précipitation de carbonitrures. De tels aciers sont susceptibles d'un fort durcissement par écrouissage, en particulier par tréfilage (Fig. 2), permettant leur utilisation comme fils à haute résistance.

\subsubsection{Aciers dual-phase}

Afin d'obtenir des aciers présentant un niveau de résistance supérieur, il est possible de refroidir plus rapidement l'alliage lorsque l'on a atteint une fraction volumique suffisante de constituant ferritique. L'austénite résiduelle se transforme alors en bainite ou en martensite, selon sa composition au moment de la trempe et en fonction de la vitesse locale de refroidissement et de la température de fin de refroidissement. Le schéma de refroidissement et la composition sont ajustés de façon à pouvoir faire varier la fraction volumique de seconde phase. La morphologie des phases et leur répartition (Fig. 4) peuvent être également fortement modifiées [13], jouant directement sur les propriétés mécaniques de l'acier.

Ces structures se rencontrent le plus souvent dans les aciers de construction mécanique, pour les pipe-lines, ou pour la construction navale. La microstructure est alors adaptée de façon à présenter une ténacité élevée, même à basse température. Actuellement, ces aciers trouvent un nouveau développement dans la construction automobile permettant ainsi une diminution d'épaisseur à résistance constante, donc un allègement potentiel. 

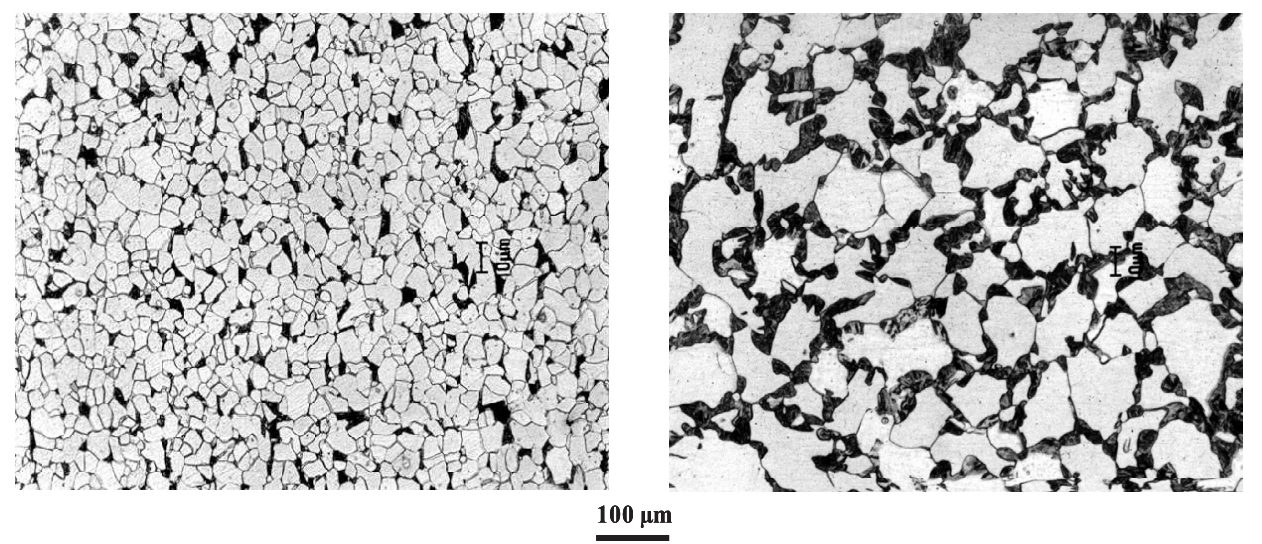

Fig. 4. Exemples de structure biphasée ferrite-martensite (ferrite en clair) : la composition initiale de l'alliage est similaire, seul le schéma de refroidissement permet de faire varier la fraction volumique. On note 2 cas extrêmes : soit des îlots de martensite dispersés dans la matrice ferritique, soit un squelette quasi-continu de martensite.

\subsubsection{Aciers TRIP}

Pour certaines compositions (en général, ajouts de silicium, d'aluminium et de manganèse), un palier de maintien à température moyenne lors du refroidissement permet d'enrichir suffisamment l'austénite résiduelle pour la rendre stable à température ambiante. Ces îlots d'austénite résiduelle peuvent se transformer en martensite lors de la mise en forme de l'acier. On dispose ainsi d'un acier biphasé dont les caractéristiques mécaniques de la seconde phase évoluent en cours de déformation. Ces aciers possèdent des propriétés mécaniques très intéressantes puisqu'ils combinent une résistance à la rupture élevée (supérieure à $1000 \mathrm{MPa}$ ) tout en conservant des allongements à rupture de l'ordre de $25 \%$.

Ces aciers, connus pour les applications en produits longs, deviennent une nouvelle génération d'aciers pour la construction automobile afin d'alléger les pièces de structure et de renfort. Le comportement particulier de la seconde phase durcissante confère également de bonnes caractéristiques de tenue à grande vitesse de déformation, utiles pour les pièces de sécurité devant absorber l'énergie lors de chocs [14].

\subsection{Les paramètres influents}

La microstructure finale dépend :

- de la composition initiale de l'alliage;

- de la microstructure de l'austénite avant la transformation de phase;

- du schéma de refroidissement.

Comme décrit succinctement ci-dessous, ces paramètres ne sont pas indépendants et les leviers d'action sont multiples.

\subsubsection{Composition chimique}

La composition de l'alliage intervient directement dans la nature des constituants formés et dans leur fraction volumique maximale. Comme nous venons de le voir, il est également important de prendre en compte les évolutions locales de la composition au cours de la transformation ou lors de maintiens en température : cette composition locale définit le type de transformation de l'austénite résiduelle et la dureté finale du produit de transformation.

Les ségrégations héritées de la solidification (partage des éléments entre les phases liquide et solide) peuvent donner lieu, dans les aciers à moyenne teneur en carbone, à des structures en bandes (Fig. 5). Des zones carburées et riches en éléments d'alliage alternent avec des domaines faiblement alliés [15]; sous l'effet du laminage à chaud, ces zones forment des couches parallèles au plan de la tôle [16]. Ces hétérogénéités de structure sont en général néfastes car elles ne permettent pas d'optimiser les propriétés mécaniques moyennes du produit et elles génèrent des zones dures et souvent fragiles, réduisant à la ténacité.

\subsubsection{Structure austénitique}

Dans la mesure où la transformation ferritique débute aux joints de grain de l'austénite, la taille de ces grains avant transformation est un paramètre structural de première importance. Lors du refroidissement, pour une fraction volumique de ferrite donnée, associée à une certaine composition de l'austénite non encore transformée, la ferrite forme un réseau plus ou moins continu selon la surface totale de joints de grain austénitique. Ceci conduit, pour une même composition de l'alliage, aux différences de structure observées dans la figure 4 [13].

Lors de l'élaboration, l'austénite est généralement déformée à chaud par laminage avant transformation. Ainsi, l'état structural de l'austénite joue également un rôle important dans la nature et dans la taille des constituants formés. Selon les cas, l'austénite peut être écrouie, restaurée ou recristallisée; souvent ces différents états se retrouvent simultanément dans le même produit en fonction de l'histoire thermomécanique locale et des éventuelles ségrégations. 


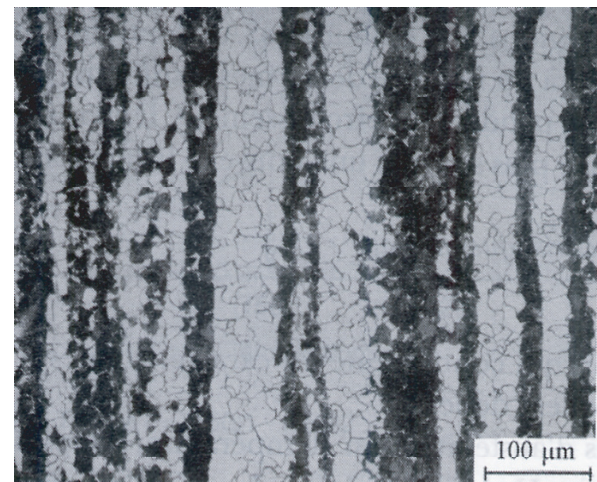

a)

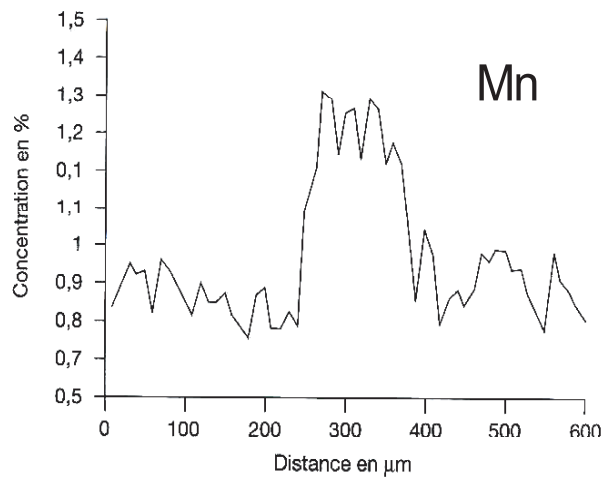

b)

Fig. 5. Aspect micrographique d'une structure ferrito-perlitique en bandes (a). Cette structure est due à l'existence de zones ségrégées dans l'épaisseur de la tôle; cette ségrégation est mise en évidence, par exemple, par la distribution du manganèse (b).

Les structures de déformation de l'austénite tendent à affiner les microstructures des constituants formés (taille de grain ferritique, taille de lattes de martensite ou de bainite, ... ) et retardent la formation des phases de trempe.

\subsubsection{Schéma de refroidissement}

Comme nous l'avons vu, la vitesse de refroidissement a un effet direct sur la nature des constituants obtenus après transformation. De plus, combiné à différents états microstructuraux de l'austénite après le laminage à chaud, le schéma de refroidissement permet de faire varier l'état de biphasage du produit final. Un maintien en température pendant le refroidissement permet d'enrichir l'austénite par diffusion afin de la stabiliser pour rechercher un effet TRIP, par exemple. Les nouvelles voies de production des aciers multiphasés utilisent pleinement ces possibilités de façon à obtenir les propriétés visées; il s'agit du laminage à température contrôlée.

\subsection{Le comportement mécanique}

La description du comportement mécanique d'un alliage biphasé ou multiphasé nécessite la connaissance

- de la microstructure de l'alliage : nature des constituants, taille et forme des éléments microstructuraux, répartition des constituants, ...

- du comportement individuel de chaque constituant.

Le premier point a été détaillé dans les paragraphes précédents. On peut obtenir ces informations par observations métallographiques quantitatives. Les modélisations métallurgiques permettront de les prévoir avec de plus en plus de précision.

\subsubsection{Comportement ferritique}

Le comportement de la ferrite peut être décrit assez simplement dans la mesure où il ne dépend que d'éléments microstructuraux « élémentaires » comme la taille de grain ou l'état de précipitation. Une loi à bases physiques a été développée pour les nuances ferritiques à partir des travaux de Kocks, de Mecking et de Estrin (voir [17]). La contrainte d'écoulement est proportionnelle à la racine carrée de la densité de dislocations :

$$
\sigma_{\alpha}=\sigma_{\alpha_{o}}+\alpha \mu b \sqrt{\rho}
$$

En considérant que l'évolution de la densité de dislocations résulte d'un terme de stockage, fonction de la taille de grain $D$, et d'un terme d'annihilation :

$$
\frac{\mathrm{d} \rho}{\mathrm{d} \varepsilon}=\frac{k}{b D}-f \rho
$$

il est possible d'exprimer l'évolution de la contrainte en fonction de la déformation :

$$
\sigma_{\alpha}=\sigma_{\alpha_{o}}+\frac{\alpha \mu \sqrt{b k}}{\sqrt{D}} \sqrt{\frac{1-\exp (-f \varepsilon)}{f}}
$$

où $\sigma_{\alpha_{o}}$ intègre les effets de durcissement des éléments en solution solide et de la précipitation [18].

Il a été montré, pour une large gamme de nuances ferritiques, que le comportement mécanique en traction uniaxiale pouvait être décrit par un seul couple des paramètres $k$ et $f[17,18]$.

\subsubsection{Nuances ferrito-perlitiques}

Le comportement des nuances ferrito-perlitiques est alors décrit de façon phénoménologique par une loi des mélanges à partir des équations (1) et (4). Les paramètres structuraux sont la taille de grain ferritique, l'espacement interlamellaire de la perlite, et la fraction volumique de perlite. À partir de la loi des mélanges suivante :

$$
\sigma(\varepsilon)=\left(1-F_{p}\right) \sigma_{\alpha}\left(\varepsilon_{\alpha}\right)+F_{p} \sigma_{p}\left(\varepsilon_{p}\right)
$$

la comparaison entre les prévisions et les mesures donne un accord satisfaisant pour un large domaine de fraction volumique de perlite $F_{p}$ en supposant, en première 


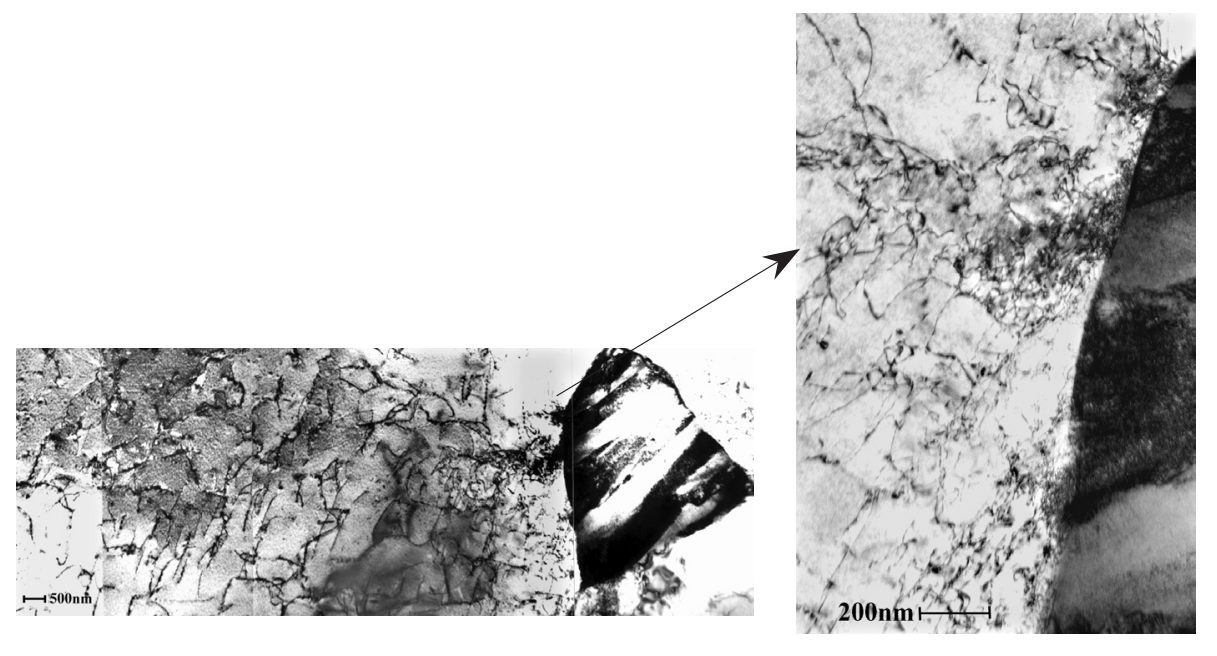

a)

b)

Fig. 6. Dislocations observées dans la ferrite au voisinage d'un îlot de martensite (en sombre sur la micrographie) et créées par la transformation de phase avec changement de volume. (b) détail de l'interface martensite-ferrite.

approximation, un rapport constant entre $\varepsilon_{\alpha}$ et $\varepsilon_{p}$, et égal à 3. Ce rapport est un paramètre d'ajustement et les améliorations possibles de ces lois passent par une meilleure description mécanique du comportement des matériaux biphasés. Une proposition récente [19] permet une approche simplifiée à partir de l'équivalence des énergies de déformation dans les différentes phases au cours de la déformation. Elle pourrait représenter une approximation de calculs mécaniques plus complets de type homogénéisation.

\subsubsection{Influence de la transformation martensitique}

Dans le cas des nuances ferrito-martensitiques, la transformation de l'austénite résiduelle se produit avec un changement de volume. Ceci induit une déformation dans la matrice ferritique environnante. Cette déformation est visualisée par la présence de dislocations au voisinage des îlots de martensite formés (Fig. 6). La densité de dislocations locale, mesurée ou estimée à partir du changement de volume induit, est de l'ordre de $10^{13} \mathrm{~m}^{-2}$ [13].

Ce pré-écrouissage local accroît légèrement la limite d'élasticité et peut modifier le comportement en supprimant, par exemple, le palier de Lüders en début de déformation. Sa prise en compte est nécessaire à une modélisation du comportement mécanique de la structure biphasée.

\subsubsection{Durcissement par martensite et bainite}

En plus de l'effet de plasticité locale induite évoqué ci-dessus, la description du comportement des nuances ferrito-martensitiques et ferrito-bainitiques pose le problème de la prise en compte du comportement des phases de trempe.
D'une part, ces constituants sont formés de petites plaquettes allongées dont une des dimensions est relativement faible, de l'ordre du micromètre. Les interfaces entre les lattes présentent également une forte densité locale de dislocations et d'éventuelles précipitations de cémentite. D'autre part, la microstructure de ces constituants est fortement liée à la transformation de phase, c'est-à-dire varie en fonction de la fraction volumique formée, pour une même composition de l'alliage.

En plus des difficultés liées à une bonne prise en compte de la morphologie et de la distribution de ces constituants, on voit donc une seconde difficulté liée à la détermination même de leur comportement local. La mesure directe est impossible, le comportement d'un matériau complètement martensitique ou bainitique ayant toute chance d'être différent (voire fragile). Seules des mesures de microdureté locales peuvent donner quelques éléments, mais le comportement plastique reste difficile à atteindre.

D'un point de vue pratique, on se limite le plus souvent à l'utilisation d'une loi des mélanges en considérant que le comportement de la martensite ou de la bainite est parfaitement plastique et que la contrainte est une fonction de la teneur locale en carbone. Des progrès doivent être faits par une meilleure compréhension de la déformation de ces structures en lattes et/ou par résolution du problème inverse à partir de la connaissance expérimentale du comportement de l'alliage et du comportement de la ferrite exprimé par la relation (4).

Le comportement des aciers TRIP est encore compliqué par la prise en compte de la transformation induite de l'austénite résiduelle. Cette transformation est certainement modifiée par l'état de contrainte local (en particulier la pression hydrostatique) et par la taille et la morphologie des îlots d'austénite ${ }^{3}$. C'est également un

\footnotetext{
${ }^{3}$ Une diminution de la taille des îlots d'austénite semble retarder la transformation martensitique.
} 
large champ d'étude dans la mesure où la cinétique de transformation martensitique au cours de la déformation a une influence directe sur l'allongement réparti de ces nuances.

Enfin les développements actuels d'aciers à très haute résistance conduisent à la production d'aciers à structure complexe, composée de ferrite, de bainite, et d'îlots d'austénite résiduelle. De plus, les tailles de chaque constituant sont affinées et avoisinent le micromètre afin de bénéficier au maximum de l'effet de durcissement de type Hall-Petch. La prévision du comportement mécanique de ces aciers (au moins de leur limite d'élasticité et de leur résistance à la rupture) reste un défi.

\section{Conclusions}

Nous avons vu que les spécificités de la métallurgie des aciers permettent l'obtention d'une gamme variée de nuances biphasées ou multiphasées. Dès à présent, en ajustant la composition chimique et les paramètres d'élaboration, il est possible de produire des aciers à haute ou très haute résistance dont l'utilisation s'accroît fortement dans l'industrie automobile.

Le comportement mécanique de ces aciers, mesuré expérimentalement pour chaque nuance et chaque type de microstructure, ne fait encore l'objet que de modélisations simples et toujours a posteriori. Le développement de l'utilisation de ces aciers pour de nouvelles pièces et de nouvelles applications requiert des efforts importants de modélisation du comportement mécanique à partir de la seule connaissance de la microstructure. Ces modélisations posent encore de nombreux problèmes tels que la prise en compte correcte de la morphologie des phases durcissantes et de leur répartition.

Enfin, les caractéristiques mécaniques doivent s'entendre au sens large, c'est-à-dire prendre en compte la description du comportement en sollicitation multiaxiale, et également la tenue au choc et le comportement en fatigue aléatoire à grand nombre de cycles.

Remerciements. L'auteur remercie P. Buessler (IRSID), J. Drillet (IRSID), N. Guelton (ARCELOR), et B. Proust (CRI) pour l'aide apportée à la préparation de cette présentation et pour l'utilisation de certains de leurs résultats.

\section{Références}

[1] D.S. Zhou, G.J. Shiflet, Ferrite-cementite crystallography in pearlite, (1992) Metall. Trans. A 23A (1992) 1259-1269

[2] P. Leduc, M. Janecek, E. de Courcy, D. Bouleau, J. Languillaume, N. Guelton, communication privée, IRSID, 1998
[3] X. Sauvage, N. Guelton, D. Blavette, Microstructure evolutions during drawing of a pearlitic steele containing 0.7 at.\% Copper, Scripta Mater. 46 (2002) 459-464

[4] M. Janecek, F. Louchet, B. Doisneau-Cottignies, Y. Bréchet, N. Guelton, Specific dislocation multiplication mechanisms and mechanical properties in nanoscaled multilayers: The example of pearlite, Phil. Mag. 80 (2000) $1605-1619$

[5] B. Pickering, The physical metallurgy and design of steels, Applied Sciences, Barking, 1978

[6] B. Bonnefois, J.P. Bouichou, G. Brethenoux, J. Demurger, G. Flament, V. Gréjon, B. Lapostolle, P. Sécordel, La mise en forme à froid, in : Les Aciers Spéciaux, Ch. 30, Lavoisier Tec \& Doc, 1997, pp. 921-987

[7] R.E. Reed-Hill, Physical Metallurgy Principles, Ch. 16 (Deformation Twinning and Martensite Reactions), Litton Educational Publishing, 1973, pp. 611-660

[8] J. Philibert, A. Vignes, Y. Bréchet, P. Combrade, Métallurgie : du minerai au matériau, Ch. 5, Masson, 1998, pp. 645-673

[9] B. Thomas, M. Guttmann, Transformations et structures, in : Le Livre de l'Acier, Ch. 6, Lavoisier Tec \& Doc, 1994, pp. $93-166$

[10] B. Thomas, J.H. Schmitt, Durcissement des aciers, Techniques de l'ingénieur, M4 341, 2002, pp. 1-20

[11] A. Lambert, J. Drillet, T. Sturel, A.F. Gourgues, A. Pineau, Microstructure of Martensite-Austenite Constituents in heat affected zones of high strength low alloy steel welds in Relation to toughness Properties, Sci. Technol. Welding Joining 5 (2000) 168-173

[12] J. Cahn, Acta Metal. 4 (1956) 449

[13] P. Buessler, O. Bouaziz, T. Iung, Modelling of mechanical properties and local deformation of high strength multiphase steels, ECSC Final Report, 2001

[14] J.L. Thirion, T. Hourman, D. Cornette, Press forming of dual phase high strength steels for autobody structural members parts, 40th Mechanical Working and Steel Processing Conference Proc. (Iron and Steel Society/AIME) 36 (1998) 35-51

[15] G. Auclair, A. Badard, J. Bellus, M. Guttmann, T. Iung, A. Kirsch-Racine, H. Michaud, M.T. Perrot-Simonetta, Méthodes de caractérisation, in : Les Aciers Spéciaux, Ch. 10, Lavoisier Tec \& Doc, 1997, pp. 335-376

[16] P. Corneiller, Les traitements thermiques, in : Les Aciers Spéciaux, Ch. 33, Lavoisier Tec \& Doc, 1997, pp. 1027-1059

[17] H. Petitgand, H. Réglé, O. Bouaziz, T. Iung, S. Barrois, Modeling of grain growth and mechanical properties of IF steels, in : Proc. ISS Conf. IF Steels 2000, (Pittsburgh, USA), Pub. Iron \& Steel Society, 2000, pp. 339-346

[18] O. Hamart, T. Iung, S. Lanteri, Study of Mechanical Properties and Precipitation in High Strength IF Steels, 40th Mechanical Working and Steel Processing Conference Proc. (Iron and Steel Society/AIME) 36 (1998) 189-194

[19] O. Bouaziz, P. Buessler, Mechanical behaviour of multiphase materials: An intermediate mixture law without fitting parameter, Revue de Métallurgie - CIT 99 (2002) $71-77$ 